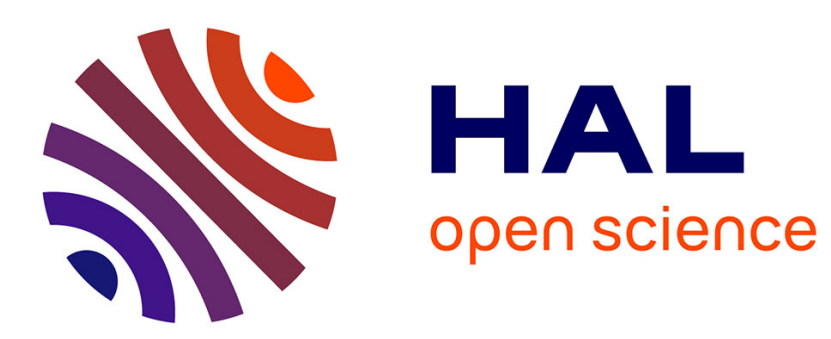

\title{
Modeling of demagnetization processes in permanent magnets measured in closed-circuit geometry
}

\author{
J. Fliegans, O. Tosoni, Nora M Dempsey, G. Delette
}

\section{To cite this version:}

J. Fliegans, O. Tosoni, Nora M Dempsey, G. Delette. Modeling of demagnetization processes in permanent magnets measured in closed-circuit geometry. Applied Physics Letters, 2020, 116 (6), pp.062405. 10.1063/1.5134561 . hal-03084028

\section{HAL Id: hal-03084028 \\ https://hal.science/hal-03084028}

Submitted on 20 Dec 2020

HAL is a multi-disciplinary open access archive for the deposit and dissemination of scientific research documents, whether they are published or not. The documents may come from teaching and research institutions in France or abroad, or from public or private research centers.
L'archive ouverte pluridisciplinaire HAL, est destinée au dépôt et à la diffusion de documents scientifiques de niveau recherche, publiés ou non, émanant des établissements d'enseignement et de recherche français ou étrangers, des laboratoires publics ou privés. 


\title{
Modelling of demagnetization processes in permanent magnets measured in closed-circuit geometry
}

\author{
J. Fliegans ${ }^{1,2}$, O. Tosoni ${ }^{1}$, N. M. Dempsey22, G. Delette1, \\ ${ }^{1}$ Univ. Grenoble-Alpes, CEA, LITEN, DTNM, LMCM, F-38000, Grenoble, France \\ 2Univ. Grenoble-Alpes, CNRS, Grenoble INP, Institut Néel, 38000 Grenoble, France
}

\begin{abstract}
$\underline{\text { Abstract }}$
The hysteresis loops of nucleation-type magnets made of exchange-decoupled grains (i.e. sintered Nd-Fe-B magnets) reflect the discrete character of magnetization switching in such materials. Due to this discrete character, the experimental determination of coercivity depends on the measurement protocol. Finite element modelling allows to investigate how the pattern of reversed grains develops during sample demagnetization performed under closed-circuit conditions, provided that the basic features of the hysteresigraph are known. Numerical modelling provides a quantitative understanding of the collective effects which are very pronounced in the closed-circuit configuration and shows how they affect both the slope of the demagnetizing curve and the sample coercivity. With a grain coercive field standard deviation adjusted to $0.1 \mathrm{~T}$, it is numerically found that the difference in coercivity between closed- and open-circuit configurations is $40 \mathrm{kA} / \mathrm{m}$, in good agreement with previous experimental data.
\end{abstract}

\section{Introduction}

Demagnetization of hard ferromagnets is intricately dependent on their microstructure. In high-performance sintered Nd-Fe-B magnets, grains are mostly exchange-decoupled and magnetization reversal spreads over the whole polycrystalline material by switching of individual grains. Basically, the polarization of a given grain is reversed when the local magnetic field exceeds the grain coercivity [1-5]. As a consequence, a pattern of reversed and non-reversed grains that are magnetostatically coupled, develops as long as demagnetization proceeds. It has been proposed in a previous work [5] that the discrete pattern should be governed by the minimization of the magnetostatic energy. Such effects are of importance since they could lead to some misinterpretation of the demagnetization

\footnotetext{
*Corresponding author : gerard.delette@cea.fr
} 
curves usually measured on magnets for system design purposes. Differences in the coercivity values measured in open-circuit and closed-circuit geometries were attributed to protocol-dependent collective effects [5]. In this work, a numerical simulation of the demagnetization of hard grains in 3D assemblies has been performed in order to analyze features of the reversal pattern and to assess the previous formalism. Open-circuit demagnetization has previously been simulated $[2-4,6]$. The analytical expression of the field produced by a rectangular prism in free space is used in [2,4] to take into account the contribution of each grain, while the dipolar approximation is employed in [6]. A finite element computation of the magnetic field has been implemented here to extend the simulation to the closed-circuit geometry. Besides allowing a comparison of open- and closed-circuit loops, multi-grain simulation enables investigation of magnetostatic coupling effects in heterogeneous magnets with property gradients.

\section{$\underline{\text { Numerical method and results }}$}

The polycrystalline magnet material is depicted in the model as a regular array of identical cubic grains of side length $10 \mu \mathrm{m}$. Each grain is assumed to be homogeneously magnetized along its easy axis and able to switch between the initial positive value $+M_{s}$ to the negative value $-M_{s}$. Reversal occurs in a given grain when the projection of the local magnetic field $\mathrm{H}$ along the $\mathrm{z}$ axis, averaged over the grain volume, exceeds the coercive field assigned to the grain $H_{c}$. A Gaussian distribution of the coercivity is ascribed to the individual grains of the array. At the beginning of the simulation, all the grains are magnetized along the $+z$ direction, yielding the remanent state reached after saturation. An external magnetic field is progressively applied on the magnet in the opposite direction by small increments $\Delta H$. After each field increment, the reversal condition is tested on all grains. The grains for which the condition for reversal is fulfilled are identified and at the end of the screening, the magnetization of these grains is switched simultaneously, leading to a new grain reversal pattern. Before further increasing the field, several equilibrium sub-steps are performed: since each grain reversal changes the overall magnetostatic field, the screening of grains is repeated after each reversal pattern update until the establishment of a stable magnetization pattern. Then, the next step, with a new field increment, is performed considering the stabilized grain magnetization pattern. 
The magnetostatic problem is solved by a Finite Element (FE) commercial software (Flux $3 \mathrm{D}$, Altair) monitored by a specific Python script. Each grain is meshed with $(4 \times 4 \times 4)$ quadratic cubic elements with size refinement near the edges and corners. It has been verified that this spatial discretization of the cubic grain leads to an accurate description of the demagnetizing field: the FE simulation of an open-circuit (oc) configuration provided results close to those previously obtained with an equivalent numerical simulation developed in [2]. The demagnetizing field was computed differently in [2] by summing the individual contribution of each grain given by an analytical expression derived by Aharoni [7] and valid for isolated bodies. In [2] the summation of individual fields allows the demagnetizing field in arrays of saturated cubic grains to be accurately depicted. Considering the field in free space around each grain corresponds to the open circuit case but for closed-circuit (cc) configurations, the flux lines are canalized around the sample by the yoke of the hysteresigraph and then by the pole pieces that are made of a non-linear (saturable) magnetic material. The demagnetizing field in a given grain depends intricately on the neighboring grains and the surrounding magnetic circuit. Accordingly, in this work FE computation replaces the analytical expression for calculating the magnetostatic field in closed-circuit. The 3D FE simulation takes into account the following features of the hysteresigraph: (i) the external field is created by a coil excited by an electric current and wound around a ferromagnetic armature, (ii) the flux is canalized towards the sample thanks to vertical arms and planar poles having a large surface area compared to the sample and (iii) the magnetic field is always measured in the mid-plane of the sample using a measuring coil. Although simple, this geometrical model gives a sufficient description of the hysteresigraph frame. In order to reduce the size of the model, three planes of symmetry $(z=0, x=0$ and $y=0)$ are considered allowing only $1 / 8$ of the total volume to be actually simulated. In the following, the simulations are labelled with the number of grains used in each direction of the reduced model, i.e. $\mathrm{n}_{\mathrm{x}} \times \mathrm{n}_{\mathrm{y}}$ $\times \mathrm{n}_{\mathrm{z}}$

The closed- and open-circuit demagnetizing curves are plotted in Fig. 1 for an array of $10 \times 10 \times 5$ grains. This corresponds to the aspect ratio of $\mathrm{Nd}-\mathrm{Fe}-\mathrm{B}$ samples measured in our previous study [5]. The mean value $\left\langle H_{c}\right\rangle$ and the standard deviation $\sigma_{c}$ are determined in such a way that the $M(H)$ curves obtained after simulation of demagnetization in closed-circuit (red solid line) fit the experimental closed-circuit demagnetization curves (red dots). The closed-circuit demagnetizing curve is found to be more rectangular than 
the coercivity distribution, which is explained by the expansion scheme of reversal resulting from magnetostatic coupling. 3D grain reversal patterns plotted in Fig. 2 show that demagnetization starts from the least coercive grains and tends to form clusters of reversed grains. Such clusters expand by aggregating more and more grains as the demagnetizing field exerted by the clusters on the neighboring grains is stronger.

The open-circuit simulation exhibits different characteristics as seen in Fig. 2. In this case, reversal begins in the grains where the cumulative effect of a low coercivity and a high local demagnetization field is critical, i.e. where the quantity $\left[H_{c} / M_{S}-N_{z}\right]$ is the lowest. The last term $N_{z}$ represents the local demagnetizing factor calculated at the first step when $H_{\text {ext }}=0 . N_{z}$ depends on the shape ratio of the magnet but it is not uniform in the magnet, as previously reported for rectangular samples [8]. The open-circuit demagnetization curve evolves almost quasi-linearly with few expansion phases. The simulated pattern of grain reversal is notably different compared to closed-circuit and exhibits more diffuse structures and quasi-homogeneous distribution of reversed grains (cf. Fig. 2 for $M / M_{S}=0$ ).

It has to be pointed out that once the grain coercivity parameters were adjusted to account for the closed-circuit experimental curve, the simulated $M(H)$ open-circuit curve followed the experimental one without any parameter adjustment. Interestingly, the difference in coercivity obtained numerically between open- and closed-circuit configurations $(\sim 40 \mathrm{kA} / \mathrm{m})$ is close to the experimental value, but larger when the latter is corrected for viscosity effects ( $\sim 25 \mathrm{kA} / \mathrm{m}$ from [5]). Finally, it has been found that this difference increases (from $\sim 40 \mathrm{kA} / \mathrm{m}$ to $\sim 60 \mathrm{kA} / \mathrm{m}$ ) when the standard deviation of the grain coercivity distribution rises (from $0.1 \mathrm{~T}$ to $0.2 \mathrm{~T}$ ). All these characteristics will be discussed in the following section, by considering magnetostatic interactions between hard grains.

A third configuration has also been investigated to shed light on magnetostatic coupling effects. It consists of a "duplex" magnet made of a low coercivity Nd-Fe-B magnet $\left(H_{c}=\right.$ $1050 \mathrm{kA} / \mathrm{m}, 10 \mathrm{~mm}$ diameter and $5 \mathrm{~mm}$ height) sandwiched between two symmetrical $\mathrm{Nd}-\mathrm{Fe}-\mathrm{B}$ magnets with higher coercivity $\left(H_{c}=1500 \mathrm{kA} / \mathrm{m}, 10 \mathrm{~mm}\right.$ diameter and $10 \mathrm{~mm}$ height). Both regions have the same remanence (1.3 T).

This stack is simulated in the closed-circuit testing condition using arrays with the same shape ratio as the individual magnets. Experimental and simulated demagnetization curves plotted in Fig. 3 show three main stages: (i) a plateau that extends up to the $H_{k}$ 
value taken at the first 'knee' of the $M(H)$ curve, resulting from a linear combination of the curves of the individual magnets, (ii) a quasi-linear decrease in $M$ and (iii) an abrupt loss of magnetization ending at the coercivity of the "duplex" magnet. The simulated curve obtained with values of $M$ and $H$ extracted at the mid-plane, which corresponds to the position of the measuring coil, is in good agreement with the experimental one, contrary to the case where the simulated curve is plotted with the average values of $M$ and $H$. Grain reversal patterns reported in Fig. 3 indicate that the second stage (ii) can in turn be decomposed into two steps. In a first step (A-D), reversal begins in the less coercive central magnet, its evolution shows few rapid expansion steps and the demagnetizing curve is found to be broader than the experimental curves of the individual magnets. Then, in a second step (E-F), reversal enters into the high coercivity region where grain switching occurs for field values lower than expected from the coercivity distribution. At the end of the second stage, expansion of clusters occurs in the more coercive magnets (F) and finally, the overall coercivity of the sample is lower than the value that would be obtained by a simple linear combination of the demagnetizing curves of all three magnets.

\section{$\underline{\text { Discussion }}$}

A formalism for the demagnetizing field has been established [9] for polycrystalline hard magnets considering the contributions of: (i) the self-demagnetizing field of the magnet, (ii) the so-called cavity field and (iii) the grain self-demagnetizing field. The two first contributions depend on the demagnetized state of the magnet; the first one depends only on the average polarization $\langle M>$ taken over the sample whereas the second one is intricately related to the magnetization pattern existing in the material. Since the grains are considered to be exchange-decoupled and made of a hard ferromagnetic phase, their polarization remains constant before reversal, as does the grain self-demagnetizing field (=- $N_{g} M_{s}$ where $N_{g}$ stands for the grain demagnetizing factor).

The cavity field reflects magnetostatic interactions between a given grain and the surrounding grains. In the previous study [5], the cavity field was expressed as a linear combination of a "homogeneous" term, proportional to the average polarization $M$, and a "collective" term proportional to $M_{s}$, that accounts for the effect of the adjacent and nonreversed grains. 


$$
H_{D}^{c a v}=(1-\alpha) \beta^{\text {hom }} N_{g} M+\alpha \beta^{c o l} N_{g} M_{S}
$$

The parameter $\alpha$ gives the weight of the collective effects in the cavity field. Note that when the parameter $\alpha$ is equal to 0 the formalism leads to the well-known "hard demag" correction. When $\alpha$ tends to 1 the $M(H)$ curve should become more rectangular in its shape since the cavity field is not dependent on $M$. The simulation in the closed-circuit condition clearly shows that when reversed grains form large clusters, this condition for $\alpha$ is fulfilled. This is a strong argument for a formalism involving collective effects. Basically, the collective character means that the cavity field experienced by a nonreversed grain is a fraction of $+N_{g} M_{s}$.

The parameter $\beta^{\text {hom }}$ was inferred to be close to unity while the last term $\beta^{c o l}$ was assumed to be equal to 0.5 considering that, before its reversal, a grain is close to a boundary between two regions with opposite magnetization directions [5]. This is again consistent with grain reversal patterns exhibiting large clusters oriented along the z-axis.

It was also previously demonstrated [5] that collective effects have an influence on the slope of the demagnetizing curve $M(H)$ (after the knee) and also on the coercive field: both effects have been experimentally observed. Basically, when the parameter $\alpha$ increases, the slope of $M(H)$ tends to increase, as reflected by the first right-hand term of (1). Moreover, the second term, independent of $M$, which acts on the grain as a "magnetizing" field, increases at the same time. As a result, an increase in coercivity is expected. This last term is larger for the closed-circuit configuration, for which collective effects are maximal $\left(\alpha^{c c}=1\right)$, than for the open-circuit case, for which these effects were found to be weaker $\left(\alpha^{o p}=0.82\right)$.

It has to be pointed out that this unique fitting parameter $\alpha^{o p}$ accounts for both the slope of the $M(H)$ curve in open-circuit and the coercivity difference between open- and closedcircuit measurements, given by:

$$
\Delta H_{c}=\left(\alpha^{o p}-1\right) \beta^{c o l} N_{g} M_{s}
$$

While this parameter value was initially fitted to experimental results from [5], an identical value $\left(\alpha^{o p}=0.82\right)$ has been obtained in this study without making any assumptions other than that concerning a dispersion in coercivity field values $\left(\sigma_{H c}=\right.$ 
$0.1 \mathrm{~T}$ ). With this low standard deviation value, $\alpha^{\text {op }}$ is found to be close to 1 , which corresponds to a reversed grain structure that doesn't form a fully homogeneous pattern. Actually, reversed grains tend to align into axially oriented one-grain-wide columns. This is explained by magnetostatic interactions between hard grains acting as follows: since the coercive field of grains are rather close in value, once a low coercivity grain reverses it increases locally the demagnetizing field felt by the upper and lower grains, by an amount that is sufficient to trigger reversal. It is worth noting that when the standard deviation is raised from $0.1 \mathrm{~T}$ to $0.2 \mathrm{~T}$, the parameter $\alpha^{\rho p}$ is found to be lower $(=0.6)$, meaning a reduction of the weight of collective effects. Correspondingly, the simulation shows a more homogeneous pattern of grain reversal.

In the duplex magnet case simulated here in the closed-circuit configuration, it has been found that, during the first step (A-D), demagnetization spreads into the central magnet differently than it would for the fully collective scheme, which exhibits large clusters of grains. This is due to volume charges that accumulate at the interface between magnets where magnetization is discontinuous, which tend to promote multi-domain patterns. Moreover, the magnetostatic field induced by the higher coercivity grains delays demagnetization of the central magnet. However, before expansion of reversal into the central region, the demagnetizing field felt by the higher coercivity grains located at the interface becomes strong enough to trigger reversal in the upper magnet, which in turn rapidly becomes dominated by collective effects.

\section{Conclusions}

The numerical simulation of hard ferromagnetic magnets composed of exchangedecoupled grains has been implemented in this study to provide a quantitative understanding of demagnetization curves. The findings apply mainly to sintered $\mathrm{Nd}_{2} \mathrm{Fe}_{14} \mathrm{~B}$-type materials which are the most common high-performance magnets used in industry. The granular nature of this material has been explicitly taken into account to describe the behavior of an assembly of about 1000 grains. The simulation is in very good agreement with the demagnetizing field formalism proposed previously [5], in which the cavity field comprises a well-known homogeneous contribution and a second part reflecting collective effects. The latter is justified here by the development of large clusters of reversed grains, with such patterns being more prevalent in closed-circuit 
measurements than in open-circuit ones. Besides offering a quantitative explanation of the coercivity differences observed between open- and closed-circuit measurements, the model helps in understanding demagnetization processes in heterogeneous magnets. Magnetostatic interactions between low and high coercivity zones, that exist for instance in large Dy-diffused magnets, may lead to a loss of squareness of $M(H)$ curves measured in closed-circuit geometry. More generally, this study emphasizes the fact that the performance of a given magnet designed for an application should be evaluated by taking into account the real distribution of demagnetizing fields. 


\section{$\underline{\text { References }}$}

[1] M. Soderžnik, H. Sepehri-Amin, T.T. Sasaki, T. Ohkubo, Y. Takada, T. Sato, Y. Kaneko, A. Kato, T. Schrefl, and K. Hono, "Magnetization reversal of exchange-coupled and exchange-decoupled Nd-Fe-B magnets observed by magneto-optical Kerr effect microscopy," Acta Mater., vol. 135, pp. 68-76, Aug. 2017.

[2] A. N. Dobrynin, V. M. T. S. Barthem, and D. Givord, "Revisiting magnetization processes in granular hard magnetic materials," Appl. Phys. Lett., vol. 95, no. 5, p. 052511, Aug. 2009.

[3] A. N. Dobrynin, V. M. T. S. Barthem, F. Ingwiller, and D. Givord, "Influence of dipolar collective effects on coercivity and demagnetizing factors in hard magnetic materials," Phys. Rev. B, vol. 81, no. 17, May 2010.

[4] A. N. Dobrynin, T. R. Gao, N. M. Dempsey, and D. Givord, "Experimental determination of the magnetization dependent part of the demagnetizing field in hard magnetic materials," Appl. Phys. Lett., vol. 97, no. 19, p. 192506, Nov. 2010.

[5] J. Fliegans, G. Delette, A. N. Dobrynin, N. M. Dempsey, and D. Givord, "Closed-Circuit Versus Open-Circuit Characterization of Hard Magnets," IEEE Trans. Magn., vol. 55, no. 2, pp. 1-5, Feb. 2019.

[6] C. Navau, D.-X. Chen, A. Sanchez, and N. Del-Valle, "Demagnetizing effects in granular hard magnetic bodies," J. Appl. Phys., vol. 109, no. 9, p. 093901 , May 2011.

[7] A., Aharoni, Introduction to the Theory of Ferromagnetism (Oxford University Press, Oxford, 1996).

[8] D.-X. Chen, E. Pardo, and A. Sanchez, "Demagnetizing factors of rectangular prisms and ellipsoids,” IEEE Trans. Magn., vol. 38, no. 4, pp. 1742-1752, 2002.

[9] H. Kronmüller, "Theory of nucleation fields in inhomogeneous ferromagnets," Phys. Status Solidi B, vol. 144, no. 1, pp. 385-396, Nov. 1987. 


\section{Figure captions}

Fig. 1: Demagnetizing curves: experimental (lines) and simulated (dots) in closed- (red) and open-circuit (black) configurations (10x10x5 grains).

Fig. 2: D grain reversal patterns at selected demagnetization values simulated in closed(up) and open-circuit (down) configurations (10x10x5 grains). The filled cubes refer to reversed grains. The 3 visible faces of the cubic boundary correspond to the 3 planes of symmetry.

Fig. 3: Experimental demagnetizing curve (solid, red) measured by a hysteresigraph device (closed-circuit) and simulated demagnetizing curves for the duplex magnet (dotted points, red open circles = values at mid plane, black open squares $=$ averaged values). The dotted lines refer to the experimental curves of individual magnets (green for high coercivity, blue for low coercivity) and the fine dotted line (black) represents the linear combination of the measured demagnetizing curves of individual magnets. Grain reversal patterns for specified field values labelled D-E-F in mid-plane with symmetries duplicated. 


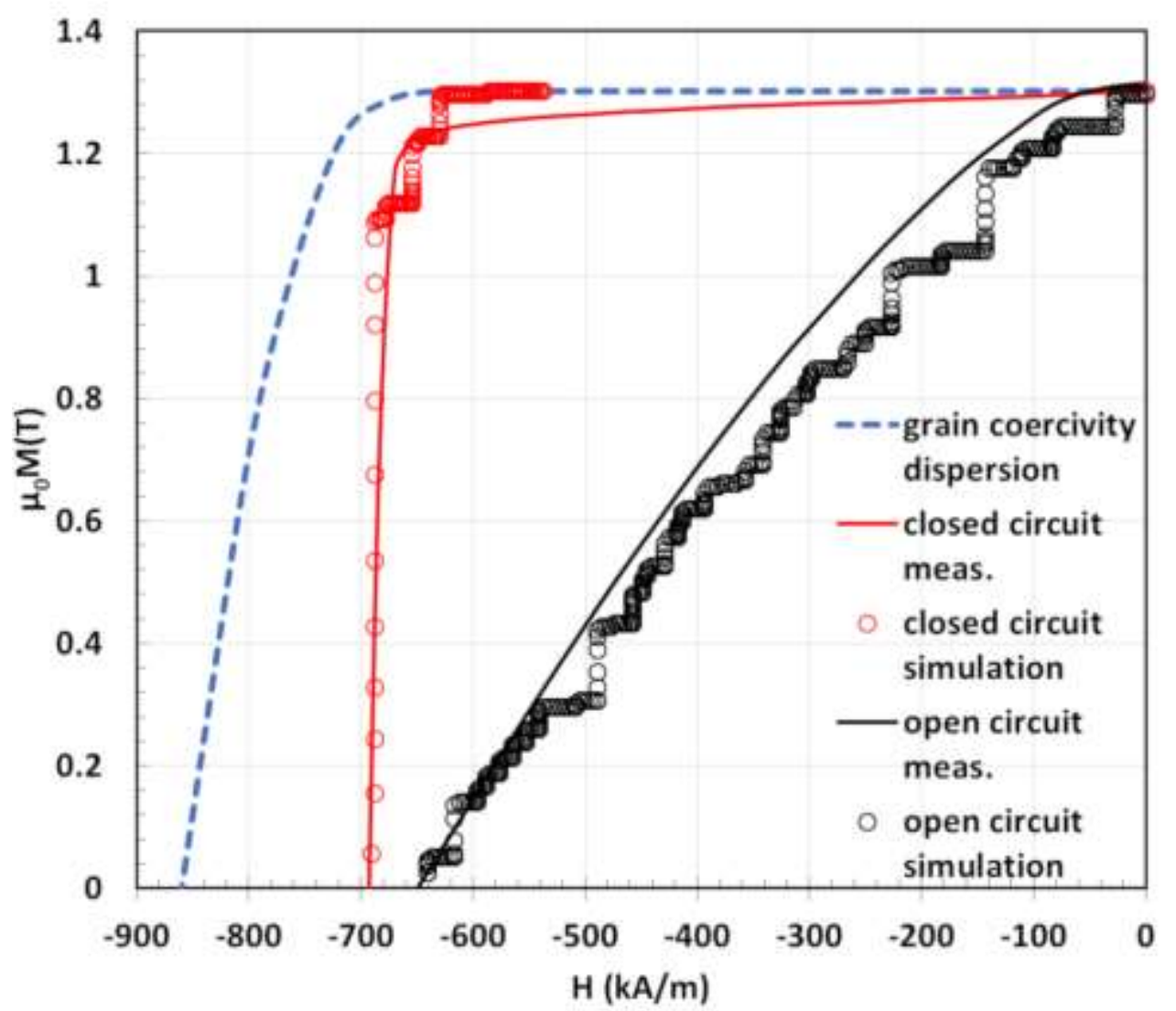

Fig. 1: Demagnetizing curves: experimental (lines) and simulated (dots) in closed- (red) and open-circuit (black) configurations (10x10x5 grains). 

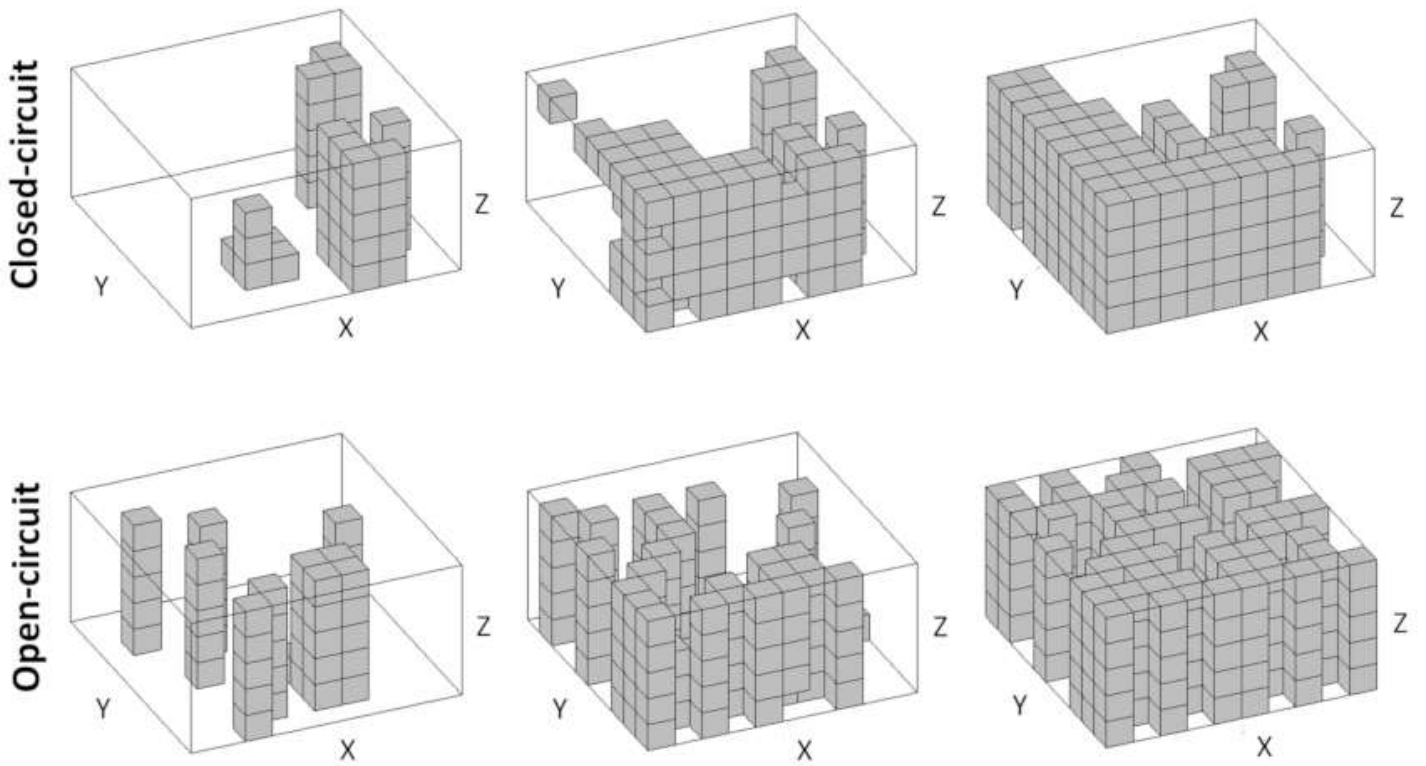

$\mathrm{M} / \mathrm{M}_{\mathrm{s}}=0.8$

$\mathrm{M} / \mathrm{M}_{\mathrm{s}}=0.5$

$\mathrm{M} / \mathrm{M}_{\mathrm{s}}=0$

Fig. 2: 3D grain reversal patterns at selected demagnetization values simulated in closed(up) and open-circuit (down) configurations (10x10x5 grains). The filled cubes refer to reversed grains. The 3 visible faces of the cubic boundary correspond to the 3 planes of symmetry. 


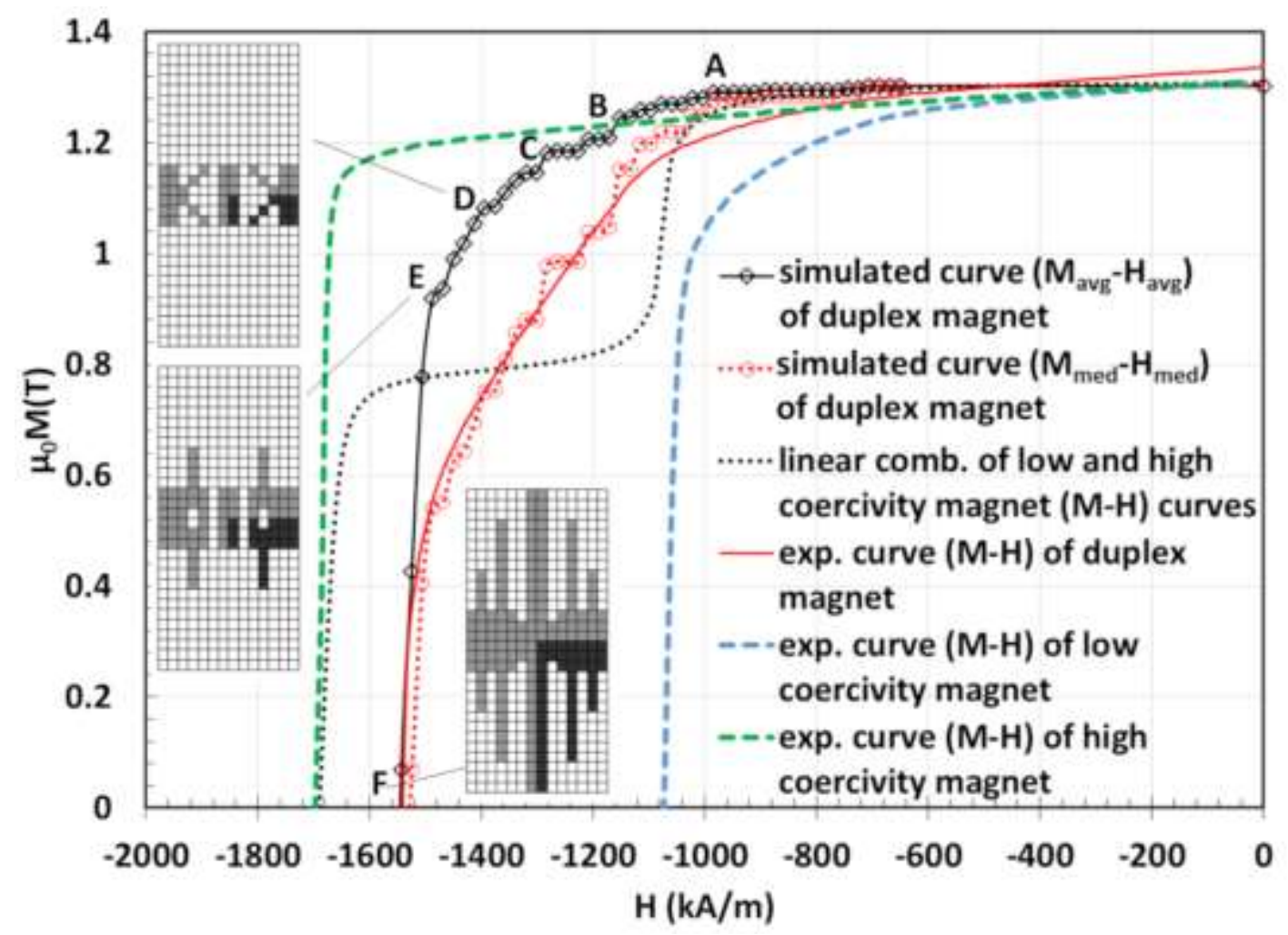

Fig. 3: Experimental demagnetizing curve (solid, red) measured by the hysteresigraph device (closed-circuit) and simulated demagnetizing curves for the duplex magnet (dotted points, red open circles $=$ values at mid plane, black open squares $=$ averaged values). The dotted lines refer to the experimental curves of individual magnets (green for high coercivity, blue for low coercivity) and the fine dotted line (black) represents the linear combination of the measured demagnetizing curves of individual magnets. Grain reversal patterns for specified field values labelled D-E-F in mid-plane with symmetries duplicated. 